\title{
A spectral coupled ocean-atmosphere model
}

R. C. Balgovind*

\author{
C. S. Frederiksen ${ }^{\dagger}$
}

J. S. Frederiksen

\begin{abstract}
A spectral coupled ocean-atmosphere model, suitable for studying the El-Nino Southern Oscillation, has been formulated. The atmospheric component is global in its extent, while the oceanic part is a hemispheric basin representing the Pacific Ocean. Multi-decadal integrations of the model have been performed with realistic topography and seasonal variations in the atmospheric radiative forcing, and for resolutions ranging from low (rhomboidal wavenumber 15 (R15)) to
\end{abstract}

* CRC for Southern Hemisphere Meteorology.

$\dagger$ Bureau of Meteorology Research Centre. mailto:c.frederiksen@bom.gov . au

$\ddagger$ CSIRO Atmospheric Research.

${ }^{0}$ See http: //anziamj . austms .org. au/V42/CTAC99/Balg for this article and ancillary services, (C) Austral. Mathematical Soc. 2000. Published 27 Nov 2000. 
intermediate (R31) to high (R60). El-Nino Southern Oscillations are produced with realistic frequency spectra, even at low resolution. The reason for this is the conserving properties of the spectral technique (in the inviscid case) and hence the model is stable with realistic and small dissipation while commonly used grid-point models require too large dissipation for stability.

\section{Contents}

1 Introduction

C185

2 Model Details

C186

2.1 Atmospheric Model . . . . . . . . . . . . . . . . C186

2.2 Oceanic Model . . . . . . . . . . . . . . . . . . . . . C188

2.3 Discretization . . . . . . . . . . . . . . . C189

2.4 Orography . . . . . . . . . . . . . . . C C191

2.5 Coupling . . . . . . . . . . . . . . . . . . . C192

2.6 Radiative Heating . . . . . . . . . . . . . . . . . . . . . C193

2.7 Dissipation and Thermal Conduction . . . . . . . . . . C194

2.8 Ocean Basin Symmetry . . . . . . . . . . . . . C194

3 Results $\quad$ C195

4 Conclusions $\quad$ C198 


\section{Introduction}

Over the last decade and a half, a large volume of literature (see, for example, [6] and the review in [5]) has been written on the phenomenon known as El Nino and the Southern Oscillation (ENSO). ENSO has a profound impact globally on the climate and consequently on the economy. The ENSO cycle is a quasi-oscillation of the coupled atmospheric-oceanic system. It arises through the interaction between the tropical ocean and the global atmosphere. The consequences of ENSO are global, but the oceanic interactions vital for its existence seems to occur only in the tropical Pacific.

Many attempts have been made to explain the large variance in the tropical sea surface temperature, at periods from 2 to 5 years, by coupling oceanic and atmospheric models. It is now understood that the inter-annual variation is a manifestation of coupled ocean-atmosphere instability. The theory (reviewed in [5]) most widely used to explain ENSO suggests that we should see evidence of both Kelvin and Rossby waves propagating within the equatorial Pacific Ocean wave guide. Rossby waves move westward and reflect at the western boundary as eastward fast moving Kelvin waves. This is the essential ingredient of the delayed action oscillator. Philander [5] suggests that the Kelvin waves should have a maximum amplitude at the equator and Rossby wave should have its maximum at around $6^{\circ}$ latitude. In this paper, 
we develop a model which can look at the mechanisms responsible for the ENSO type vacillations.

The plan of this paper is as follows, In Section 2, we describe the formulation and implementation of a spectral coupled ocean-atmosphere model of intermediate complexity and in Section 3 we describe some results at different horizontal resolution. Our conclusions are presented in Section 4.

\section{Model Details}

The model detailed here describes the dynamics in, and interactions between, a global atmosphere and an hemispheric ocean (approximating the Pacific Ocean basin). Simple coupling processes involving surface heat fluxes and wind stresses are used to couple the atmospheric and oceanic components. The models and the coupling are described briefly below.

\subsection{Atmospheric Model}

The atmospheric component of the coupled model is based on the primitive equations on a sphere and is similar to that described in [1]. The model is formulated in terms of vorticity, divergence and potential temperature. Here, we use a "dry" atmosphere and hence all latent heat effects are excluded. 
Parametrisations of friction, sensible heating, and eddy effects are included. The governing equations are,

$$
\begin{gathered}
\frac{\partial \zeta}{\partial t}=-L((\zeta+f) U,(\zeta+f) V)-L\left(\omega \frac{\partial V}{\partial p},-\omega \frac{\partial U}{\partial p}\right)-\vec{k} \cdot \nabla \times F, \\
\frac{\partial D}{\partial t}=L((\zeta+f) U,-(\zeta+f) U)-L\left(\omega \frac{\partial U}{\partial p}, \omega \frac{\partial V}{\partial p}\right)- \\
\nabla^{2} \Phi-\nabla^{2}\left(\frac{U^{2}+V^{2}}{2 \cos ^{2} \phi}\right)-\nabla \cdot F, \\
\frac{\partial \theta}{\partial t}=-L(U \theta, V \theta)-\frac{\partial(\omega \theta)}{\partial p}+\left(\frac{p_{0}}{p}\right)^{\frac{R}{c_{p}}} \frac{Q}{c_{p}} \\
\frac{\partial \omega}{\partial p}=-L(U, V), \\
\frac{\partial \Phi}{\partial p}=-\frac{R \theta}{p}\left(\frac{p}{p_{0}}\right)^{\frac{R}{c_{p}}},
\end{gathered}
$$

and,

$$
L(A, B)=\frac{1}{a \cos ^{2} \phi}\left(\frac{\partial A}{\partial \lambda}+\cos \phi \frac{\partial B}{\partial \phi}\right) .
$$

Here, $\zeta$ is the vertical component of relative vorticity; $D$ is the divergence; $\theta$ is the potential temperature; $\Phi$ is the geopotential; $t$ is time; $\lambda$ is longitude; $\phi$ is latitude; $p$ is pressure and represents the vertical coordinate; $\omega=\frac{d p}{d t}$ 
is the vertical wind component in pressure coordinates; $f=2 \Omega \sin \phi$ is the Coriolis parameter; $\Omega=7.292 \times 10^{-5} \mathrm{~s}^{-1}$ is the earth's angular speed of rotation; $U=u \cos \phi$ where $u$ is longitudinal wind component; $V=v \cos \phi$ where $v$ is the meridional wind component; $F$ represents frictional forces (see below); $p_{0}$ is pressure at sea-level; $R=287 \mathrm{JK}^{-1} \mathrm{~kg}^{-1}$ is the gas constant for air; $c_{p}=1004 \mathrm{JK}^{-1} \mathrm{~kg}^{-1}$ is the specific heat of dry air at constant pressure; $Q$ is the rate of heat addition. It is convenient to separate the wind into rotational and divergent parts such that $\zeta=\nabla^{2} \psi$ and $D=\nabla^{2} \chi$, where $\psi$ is the streamfunction and $\chi$ is the velocity potential.

\subsection{Oceanic Model}

The oceanic model has a similar structure to the atmospheric component and is formulated in terms of vorticity, divergence and temperature using the Boussinesq approximation. However, the ocean is taken to be hemispheric, covering 180 degrees of longitude $\left(108.75^{\circ}\right.$ to $\left.288.75^{\circ}\right)$ and extending from $90 \mathrm{~S}$ to $90 \mathrm{~N}$ in latitude (approximating the Pacific Ocean basin). The governing equations are,

$$
\begin{gathered}
\frac{\partial \zeta^{\prime}}{\partial t}=-L\left(\left(\zeta^{\prime}+f\right) U^{\prime},\left(\zeta^{\prime}+f\right) V^{\prime}\right)-L\left(\omega^{\prime} \frac{\partial V^{\prime}}{\partial z},-\omega^{\prime} \frac{\partial U^{\prime}}{\partial z}\right)-\vec{k} \cdot \nabla \times F^{\prime}, \\
\frac{\partial D^{\prime}}{\partial t}=L\left(\left(\zeta^{\prime}+f\right) V^{\prime},-\left(\zeta^{\prime}+f\right) U^{\prime}\right)-L\left(\omega^{\prime} \frac{\partial U^{\prime}}{\partial z}, \omega^{\prime} \frac{\partial V^{\prime}}{\partial z}\right)
\end{gathered}
$$




$$
\begin{gathered}
-\nabla^{2}\left(\frac{p^{\prime}+\rho_{0}^{\prime} g z}{\rho_{0}^{\prime}}\right)-\nabla^{2}\left(\frac{U^{\prime 2}+V^{\prime 2}}{2 \cos ^{2} \phi}\right)-\nabla \cdot F^{\prime} \\
\frac{\partial T^{\prime}}{\partial t}=-L\left(U^{\prime} T^{\prime}, V^{\prime} T^{\prime}\right)-\frac{\partial\left(T^{\prime} \omega^{\prime}\right)}{\partial z}+k \frac{\partial^{2} T^{\prime}}{\partial^{2} z} \\
\frac{\partial \omega^{\prime}}{\partial z}=-L\left(U^{\prime}, V^{\prime}\right) \\
\frac{\partial\left(\frac{p^{\prime}+\rho_{0}^{\prime} g z}{\rho_{0}^{\prime}}\right)}{\partial z}=\alpha g T^{\prime} .
\end{gathered}
$$

Here, $\zeta^{\prime}, D^{\prime}, \omega^{\prime}, F^{\prime}, U^{\prime}, V^{\prime}$ and $p^{\prime}$ have the same meaning as for the atmospheric component. $T^{\prime}$ represents the ocean temperature; $z$ is the vertical coordinate in metres; $g$ is the gravitational constant $\left(9.81 \mathrm{~ms}^{-2}\right) ; \alpha$ $\left(2 \times 10^{-4} \mathrm{~K}^{-1}\right)$ is the thermal expansion coefficient for water; $\rho_{0}^{\prime}\left(1000 \mathrm{kgm}^{-3}\right)$ is a reference density for water.

\subsection{Discretization}

Here, we discuss briefly the methods by which the continuous governing equations are discretized both spatially and temporally. Both components of the coupled model have two vertical levels corresponding to $250 \mathrm{hPa}$ and $750 \mathrm{hPa}$ for the atmosphere and $50 \mathrm{~m}$ and $150 \mathrm{~m}$ for the ocean. We assume, in both cases, a level of non-divergence midway between these levels (i.e. 500hPa and $100 \mathrm{~m}$, respectively). The lower boundary condition (that is the abyss temperature ) in the ocean has the option of having a fixed or realistic meridional 
temperature profile taken at a depth of 250 meters. All derivatives with respect to the vertical coordinate $p$ (and $z$ ) are finite differenced in the vertical, according to,

$$
\left.\frac{\partial A}{\partial p}\right|_{i}=\frac{A_{i-1}-A_{i+1}}{p_{i-1}-p_{i+1}} .
$$

Horizontally, we represent each field by a finite series of spherical harmonics. Thus,

$$
A(\lambda, \mu, t)=d(A) \sum_{m=J}^{J} \sum_{l=m}^{|m|+J} A_{l}^{m}(t) P_{l}^{m}(\mu) e^{i m \lambda},
$$

where, $\mu=\sin \phi, d(A)$ is the dimension of $A$ such that spectral coefficients $A_{l}^{m}$ are non-dimensional and $P_{l}^{m}$ is the associated Legendre polynomial of the first kind. Here, we present some results for rhomboidal wavenumber 15 (R15), 31 (R31) and 60 (R60) truncations.

Time differencing is done using the semi-implicit technique. That is we define,

$$
\frac{\partial A}{\partial t}=\frac{A^{\tau}-A^{t-1}}{\Delta t}
$$

where,

$$
A^{\tau}=\frac{A^{t+1}+A^{t-1}}{2}
$$




\subsection{Orography}

In the atmospheric component the orography enters as a vorticity and divergence source due to flow over orography [3]. Thus, for example, for the atmosphere,

$$
\begin{gathered}
\frac{\partial \zeta_{3}^{h}}{\partial t}=-\nabla\left(\frac{f h}{H_{c}} \vec{V}_{3}\right), \\
\frac{\partial D_{1}^{h}}{\partial t}=-\frac{\partial D_{3}^{h}}{\partial t}=\frac{1}{2} \nabla\left(\frac{f h \vec{k} \times \vec{V}_{3}}{H_{c}}\right),
\end{gathered}
$$

where, $H_{c}$ is a representative height scale and $h$ is the topographic height. Similar terms are used for the ocean. As a consequence, it can be shown that, with a spectral representation and this choice of topographic forcing, the total energy and enstrophy are quasi-conserved in both the atmosphere and ocean in the inviscid case and in the presence of orography. This is a unique feature of our coupled model, and allows much weaker dissipation at low resolutions than is used in current models. The use of spectral techniques also allows energy spectra to be calculated directly. However, in order to use a spectral representation in the hemispheric ocean component, some symmetry constraints need to be placed on the ocean variables (see below). 


\subsection{Coupling}

Simple coupling processes involving surface heat fluxes and wind stresses are used, based on [6] to couple the atmospheric and oceanic components. The heat flux at the ocean surface is modelled following [2],

$$
H=-\gamma\left(T_{s}-T_{1}^{\prime}\right),
$$

where, $H$ is the heat flux, $\gamma=30 \mathrm{Wm}^{-2} \mathrm{~K}^{-1}$ is the strength of coupling. $T_{s}$ is the surface air temperature (derived by linear interpolation in $\log p$ from the lowest atmospheric level to the ocean surface, see for example [6]) and $T_{1}^{\prime}$ is the temperature of the ocean at $50 \mathrm{~m}$. This modifies the mean atmospheric temperature equation

$$
\frac{\partial \bar{\theta}}{\partial t}=\cdots+\frac{g H}{p_{0} \bar{p} c_{p}},
$$

and the oceanic top layer equation

$$
\frac{\partial T_{1}^{\prime}}{\partial t}=\cdots-\frac{H}{2 \rho_{0}^{\prime} c_{o} \Delta z},
$$

where, $\bar{p}=\left(\left(\frac{p_{1}}{p_{0}}\right)^{\kappa}+\left(\frac{p_{3}}{p_{0}}\right)^{\kappa}\right) / 2, \kappa=R / c_{p} c_{o}$ is the specific heat of water and $\Delta z=50 \mathrm{~m}$.

The surface stress is assumed to be proportional to the atmospheric lower level velocity. This modifies the atmospheric lower level wind equation,

$$
\frac{\partial \vec{V}_{3}}{\partial t}=\cdots-k_{\nu} \vec{V}_{3}\left|\vec{V}_{3}\right|
$$


and the upper level oceanic current equation

$$
\frac{\partial \vec{V}_{1}^{\prime}}{\partial t}=\cdots+\frac{p_{0} k_{\nu} \vec{V}_{3}\left|\vec{V}_{3}\right|}{4 \rho_{0}^{\prime} g \Delta z},
$$

where, the proportionality constant $k_{\nu}$ is chosen to represent an e-folding time of 5 days.

\subsection{Radiative Heating}

An annually varying radiative heating term is incorporated using simple "relaxation" physics with a radiative equilibrium driving term

$$
T_{e}=302+40(\sin 2 \lambda \sin \delta G+\cos 2 \lambda \cos \delta \sin G),
$$

where, $\sin G=1$ ( $\mathrm{H}$ : half day) and $\delta=$ zenith angle. This is incorporated into the model with a relaxation $(\alpha)$ of fifteen days. Hence, for example, the mean atmospheric temperature equation is modified to,

$$
\frac{\partial \bar{\theta}}{\partial t}=\cdots+\alpha\left(\bar{\theta}_{e}-\bar{\theta}\right),
$$

where, $\theta_{e}$ is the equilibrium potential temperature derived from $T_{e}$. 


\subsection{Dissipation and Thermal Conduction}

In the atmospheric model we have incorporated a horizontal eddy diffusion in each of the prognostic equations for the vorticity and the divergence of the form $K_{2}\left(\nabla^{2} \zeta+\frac{2 \zeta}{a^{2}}\right)$ and for the potential temperature equation a term $K_{2} \nabla^{2} \theta$. For truncations R15, R31 and R60 we have used $K_{2}=2.5 \times 10^{5} \mathrm{~m}^{2} \mathrm{~s}^{-1}$, $0.75 \times 10^{5} \mathrm{~m}^{2} \mathrm{~s}^{-1}$ and $0.7 \times 10^{5} \mathrm{~m}^{2} \mathrm{~s}^{-1}$, respectively. In the oceanic model we have incorporated a horizontal eddy diffusion in each of the three prognostic equations of the form $K_{4} \nabla^{4} \zeta$ as well as a vertical diffusion of the form $K \frac{\partial^{2} \zeta}{\partial z^{2}}$. For truncations R15, R31 and R60 we have used $K_{4}=2.5 \times 10^{5} \mathrm{~m}^{4} \mathrm{~s}^{-1}$, $0.75 \times 10^{5} \mathrm{~m}^{4} \mathrm{~s}^{-1}$ and $0.7 \times 10^{5} \mathrm{~m}^{4} \mathrm{~s}^{-1}$, and $K=0.5 \times 10^{-4} \mathrm{~m}^{2} \mathrm{~s}^{-1}, 1.0 \times 10^{-4} \mathrm{~m}^{2} \mathrm{~s}^{-1}$ and $1.0 \times 10^{-4} \mathrm{~m}^{2} \mathrm{~s}^{-1}$, respectively.

\subsection{Ocean Basin Symmetry}

In order to utilize a spectral formulation and to model the land boundaries of our hemispheric ocean basin, it is necessary to place some symmetry constraints on the coupling terms. In particular, the heat flux and the meridional component of wind stress are constrained to be symmetric about the eastern boundary of the basin. Conversely, the zonal component of the wind stress is forced to be anti-symmetric. With this arrangement of the coupling terms, appropriate symmetry constraints are imposed on the ocean model variables, thus ensuring the correct circulation in the basin.Thus, for example, the absolute vorticity becomes antisymmetric and the potential 
temperature symmetric.

\section{Results}

Multi-decadal integrations of the model have been performed including realistic topography and seasonal variations in the radiative forcing. The model provides quite realistic atmospheric and oceanic circulations including their seasonal variations (not shown). Thus, for example, the ocean currents on the equator are about $1 \mathrm{~ms}^{-1}$, which is similar to the observed. These currents are much smaller in grid point models which require much stronger dissipation. Also, El-Nino Southern Oscillations (ENSOs) are produced with realistic frequency spectra even with resolutions as low as wavenumber 15 . The oscillations however have lower amplitude than observed. The reasons for the realistic ENSO fluctuations in the spectral model can be shown to be related to the improved conservation properties and the fact that the spectral model is stable with realistic and small dissipation while commonly used grid point models require too large a dissipation for stability.

Figure 1 shows the time filtered (11 month running mean) 50m ocean temperature averaged between $2 \mathrm{~N}$ to $2 \mathrm{~S}$, as a function of longitude, for the last 35 years of a 40 year run from a R31 experiment. This clearly shows that there is ENSO like variability with a period of about 4-5 years throughout the 30 years, in both the western and eastern tropical Pacific Ocean. Similar results occur for the R15 and R60 runs. 

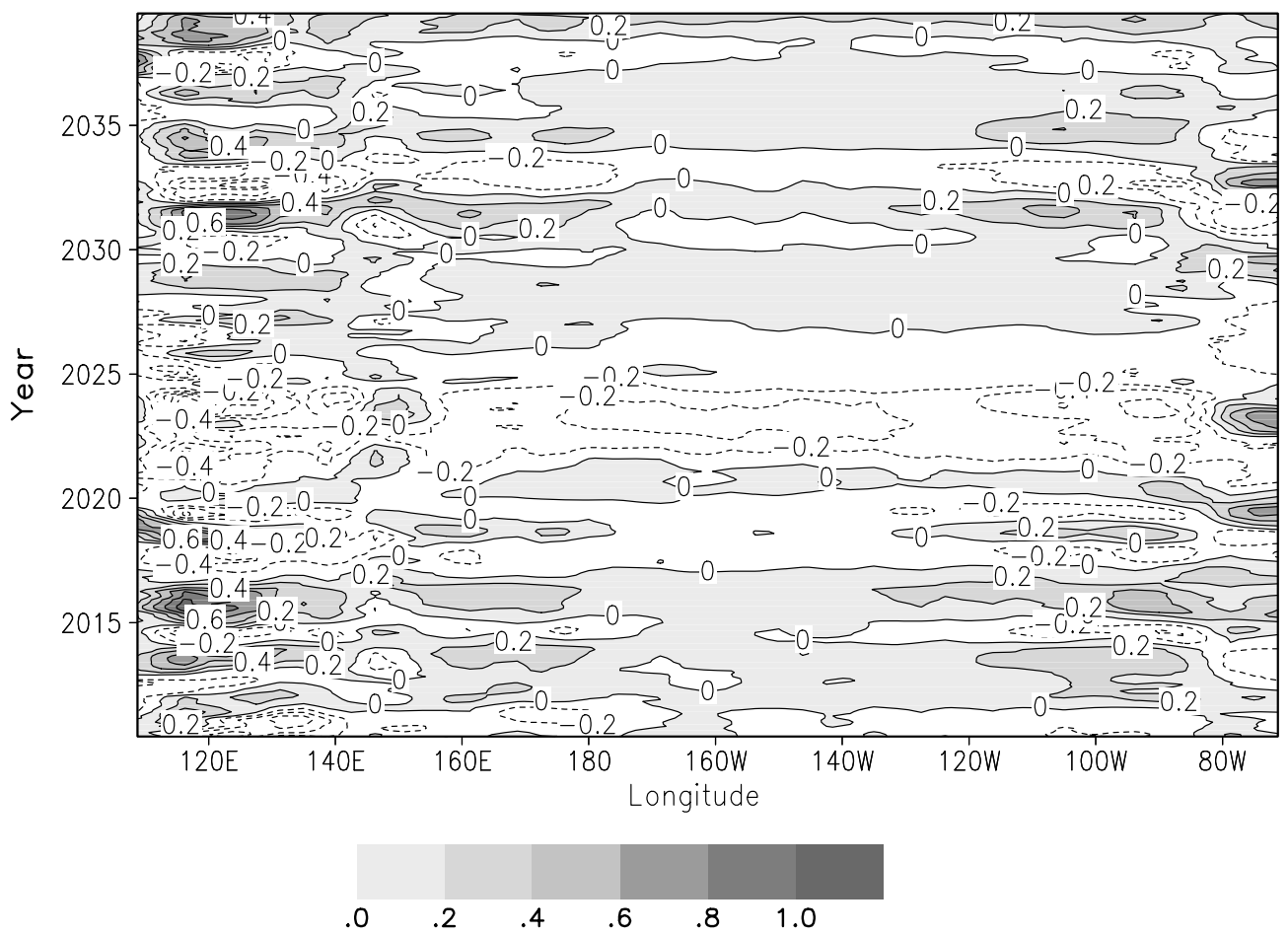

FiguRE 1: $50 \mathrm{~m}$ ocean temperature averaged over $2 \mathrm{~N}-2 \mathrm{~S}$ at $\mathrm{R} 31$ resolution 


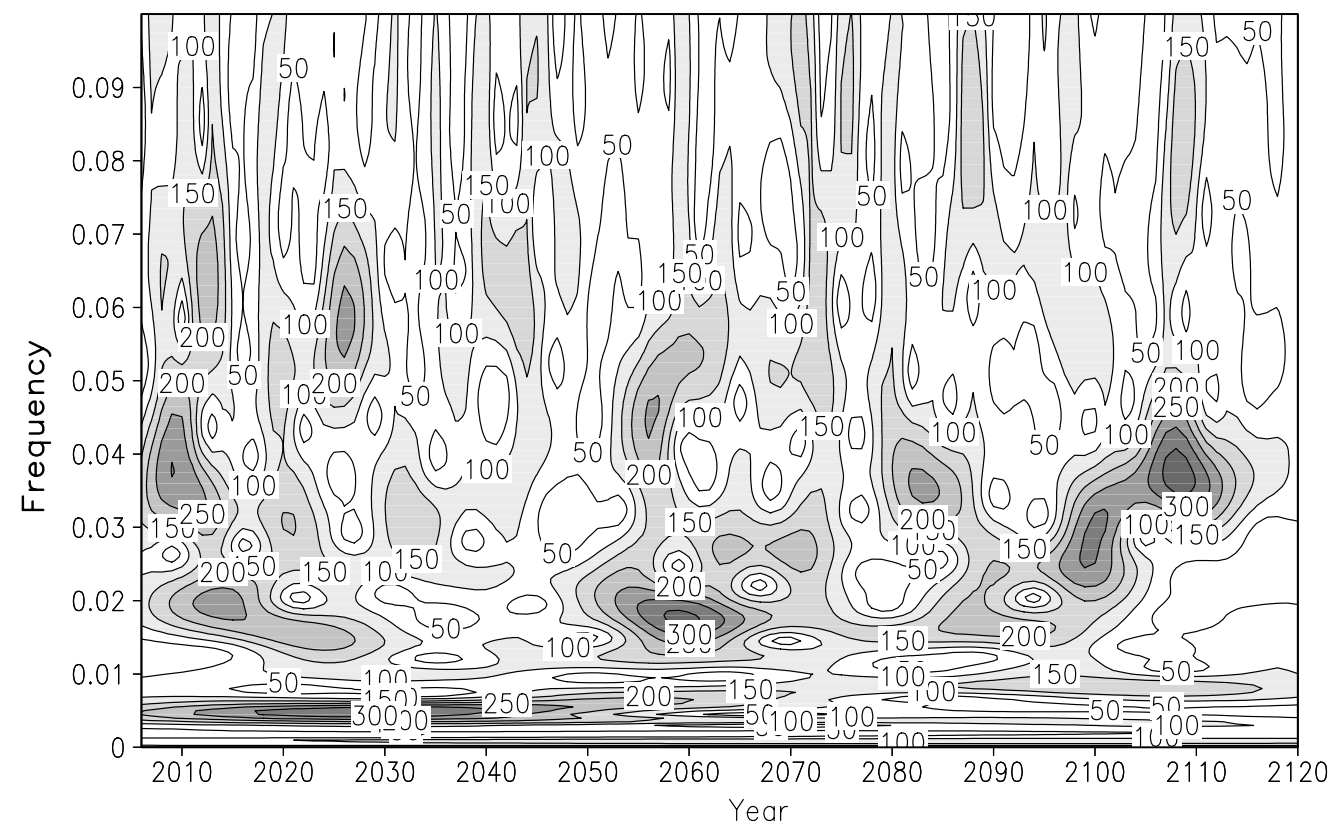

$\begin{array}{llllll}100 & 150 & 200 & 250 & 300 & 350\end{array}$

Figure 2: Wavelet analysis of the $50 \mathrm{~m}$ ocean temperature average over $10 \mathrm{~N}-10 \mathrm{~S}$ at longitude 220 and R15 resolution. 
To investigate the variability of the $50 \mathrm{~m}$ ocean temperature in the eastern part of the basin, we have conducted a wavelet analysis of the temperature averaged over (10S-10N) at 220 longitude. The results are shown in Figure 2 for a 120 year simulation at R15. The vertical axis in this diagrams represents frequency (with units per months). From this diagram, we can see variability at all different time scales. Notably, there are periods in the $2-3$ year, $4-$ 5 year and decadal time scales typically seen in the observations. This is also seen in shorter, but higher resolution, runs at R31 and R60 (not shown).

\section{Conclusions}

A spectral coupled ocean-atmosphere model, suitable for studying ENSO, has been formulated. Results from multi-decadal simulations show that the model is able to reproduce quite realistic ENSO fluctuations with realistic amplitudes and frequency spectra, even at very low resolution. A wavelet analysis shows that the model exhibits its own self-sustained variability from month to month and year to year, with spectral peaks in the 2-3 year, 45 year and decadal time scales. 


\section{References}

[1] C. S. Frederiksen and J. S. Frederiksen. North Hemisphere storm tracks and teleconnection patterns in primitive equation and quasi-geostrophic models. J. Atmos. Sci., 49:1443-1458, 1992. C186

[2] R. L. Haney. Surface thermal boundary condition for ocean circulation model. J. Phys. Oceanogr., 6:621-631, 1971. C192

[3] H. H. Hendon. Time-mean flow and variability in a nonlinear model of the atmosphere with orographic forcing. J. Atmos. Sci., 43:443-448, 1986. C191

[4] N.-C. Lau, S. G. H. Philander, and M. J. Nath. Simulation of Enso-like phenomena with a low-resolution coupled GCM of the global ocean and atmosphere. J. Climate, 5:248-307, 1992.

[5] S. G. Philander. El Nino, La Nino, and the Southern Oscillation. Academic Press, 1990. C185, C185, C185

[6] P. S. Schopf and M. J. Suarez. Vacillations in a coupled ocean-atmosphere model. J. Atmos. Sci., 45:549-566, 1988. C185, C192, C192 